\title{
Comments on "Pharmacological Characterization of Apraglutide a Novel Long-Acting Peptidic Glucagon-Like Peptide-2 Agonist for the Treatment of Short Bowel Syndrome"
}

We read with interest the publication entitled "Pharmacological Characterization of Apraglutide a Novel Long-Acting Peptidic Glucagon-Like Peptide-2 Agonist for the Treatment of Short Bowel Syndrome" (Hargrove et al., 2020). The authors investigated the pharmacology and pharmacokinetic (PK) properties of apraglutide, comparing it directly with teduglutide and glepaglutide. The authors concluded that apraglutide showed a greater ability to stimulate growth of the small intestine in rats relative to teduglutide and glepaglutide when directly compared at two doses (30 and $300 \mathrm{nmol} / \mathrm{kg}$; Fig. 5).

Given that the study results may influence future basic research, drug development programs and eventually clinical practice, we consider it important to point out some key errors relating to the synthesis and formulation of glepaglutide used by Hargrove et al. that may invalidate a number of results and conclusions in the paper.

The authors claim that glepaglutide was identified from a published patent application (WO06117565A2) and described the amino acid sequence of the peptide as well as its counter ion used in their studies (Hargrove et al. (2020), Table 1). The amino acid sequence of glepaglutide is however not correct.

Hargrove et al. prepared all peptides as trifluoroacetic acid (TFA) salts, whereas we have developed glepaglutide as an acetate salt. The salt identity of glepaglutide is of relevance for the quality of the experimental work, data generated, and validity of conclusions drawn in the paper. In addition, for the pharmacodynamic (PD) studies, apraglutide, teduglutide and low doses $(30 \mathrm{nmol} / \mathrm{kg}$ ) of glepaglutide were dissolved in $25 \mathrm{mM}$ phosphate buffer at $\mathrm{pH} 7.4$, while the high dose of glepaglutide $(300 \mathrm{nmol} / \mathrm{kg})$ was dissolved in sterile water in contrast to the other solutions.
Dissolving a TFA salt of any peptide, including glepaglutide, in an unbuffered solution will result in a solution with very low $\mathrm{pH}$. This may impact solubility and stability of glepaglutide, thus yielding potentially misleading results in PK and PD studies. Furthermore, by using a TFA salt in an unbuffered solution, we suspect that distribution from the subcutaneous injection depot is negatively impacted due to the very low $\mathrm{pH}$. Therefore, comparison of the data obtained for glepaglutide TFA salt in sterile water to the other compounds in the paper is misleading.

Given the errors in the Hargrove et al. (2020) paper, we conclude that the peptide used in the study cannot be glepaglutide. This, together with the formulation issues described above, leads us to conclude that any representations of in vivo data describing the comparison of apraglutide to glepaglutide may not be valid. The conclusion that "apraglutide has greater ability to stimulate small intestine growth relative to glepaglutide "is therefore misleading.

Research and Development, Zealand Pharma A/S,

Sydmarken 11, 2860 Søborg,

Jolanta Skarbaliene BJARNE Due LARSEN

Denmark

Mark Berner-Hansen Adam Steensberg

\section{Reference}

Hargrove DM, Alagarsamy S, Croston G, Laporte R, Qi S, Srinivasan K, Sueiras-Diaz J, Wiśniewski K, Hartwig J, Lu M, et al. (2020) Pharmacological Characterization of Apraglutide, a Novel Long-Acting Peptidic Glucagon-Like Peptide-2 Agonist, for the Treatment of Short Bowel Syndrome. J Pharmacol Exp Ther 373:193-203.

Address correspondence to: Dr. Jolanta Skarbaliene, Research and Development, Zealand Pharma A/S, Sydmarken 11, 2860 Søborg, Denmark. E-mail: JSkarbaliene@zealandpharma.com. 